\title{
Practical Inlet Boundary Conditions for Internal Flow Calculations
}

Fredrik Laurén and Jan Nordström

The self-archived postprint version of this journal article is available at Linköping University Institutional Repository (DiVA):

http://urn.kb.se/resolve?urn=urn:nbn:se:liu:diva-150377

N.B.: When citing this work, cite the original publication.

Laurén, F., Nordström, J., (2018), Practical Inlet Boundary Conditions for Internal Flow Calculations, Computers \& Fluids. https://doi.org/10.1016/j.compfluid.2018.08.011

Original publication available at:

https://doi.org/10.1016/j.compfluid.2018.08.011

Copyright: Elsevier

http://www.elsevier.com/

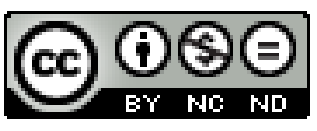




\title{
Practical Inlet Boundary Conditions for Internal Flow Calculations
}

\author{
Fredrik Laurén ${ }^{\mathrm{a}}$, Jan Nordström ${ }^{\mathrm{b}}$ \\ ${ }^{a}$ Department of Mathematics, Computational Mathematics, Linköping University, \\ SE-581 83 Linköping, Sweden (fredrik.lauren@liu.se). \\ ${ }^{b}$ Department of Mathematics, Computational Mathematics, Linköping University, \\ SE-581 83 Linköping, Sweden (jan.nordstrom@liu.se)
}

\begin{abstract}
To impose boundary conditions, data at the boundaries must be known, and consequently measurements of the imposed quantities must be available. In this paper, we consider the two most commonly used inflow boundary conditions with available data for internal flow calculations: the specification of the total temperature and total pressure. We use the energy method to prove that the specification of the total temperature and the total pressure together with the tangential velocity at an inflow boundary lead to wellposedness for the linearized compressible Euler equations. Next, these equations are discretized in space using high-order finite-difference operators on summation-by-parts form, and the boundary conditions are weakly imposed. The resulting numerical scheme is proven to be stable and the implementation of the corresponding nonlinear scheme is verified with the method of manufactured solutions. We also derive the spectrum for the continuous and discrete problems and show how to predict the convergence rate to steady state. Finally, nonlinear steady-state computations are performed, and they confirm the predicted convergence rates.
\end{abstract}

Keywords: Internal flow, inlet boundary conditions, steady state, Euler equations, well-posedness, eigenmode analysis

\section{Introduction}

Internal flow calculations are widely used in gas turbine modeling and construction of turbocharged engines [1,2]. The influence of boundary conditions on these calculations is significant and several studies have been pub- 
lished on how the boundary conditions affect these simulations $[3,4,5]$. To impose boundary conditions, data at the boundaries must be known, and consequently measurements of the imposed quantities must be available. In this paper, we consider the two most commonly used inflow boundary conditions with available data for internal flow calculations $[1,2,3,4,5,6]$ : the specification of the total temperature and total pressure (later referred to as the total variables). The two-dimensional Euler equations govern the flow, and they require three boundary conditions at a subsonic inflow boundary. We investigate if a third boundary condition can be added on to yield a well-posed initial-boundary value problem (IBVP) [7].

Solvers that rapidly converge to steady state are important in many applications $[8,9]$. Different numerical techniques such as local time-stepping and multigrid are often used to enhance the convergence rate. A requirement for these techniques to work is that the spectrum of the IBVP resides in the appropriate half of the complex plane. This requirement is also necessary for well-posedness of the problem [10]. We use the Laplace transform method and compute the spectrum of the IBVP, which determines the convergence rate to steady state $[11,12]$. See $[10,13]$ for more information about wellposedness, the energy method and the Laplace transform method.

Once well-posedness is investigated, and the continuous spectrum is derived, we proceed to the discrete approximation. High-order finite-difference operators on summation-by-parts (SBP) form are used for the discretization in space. The boundary conditions are imposed weakly using the SimultaneousApproximation-Terms (SAT) technique and the resulting linear SBP-SAT scheme is proven to be stable, using the discrete energy method [14]. Finally, we compute the discrete spectrum of the linearized IBVP and show how to predict the convergence rate to steady state for the nonlinear scheme.

The rest of the paper proceeds as follows: The governing equations and the total variables are introduced in Section 2. In Section 3, sufficient conditions for well-posedness for a general boundary operator are derived using the energy method. Later in the same section, the specific boundary conditions mentioned above are investigated. The continuous spectrum is derived in Section 4. The provably stable discrete scheme is derived in Section 5 together with the discrete spectrum. Nonlinear computations are presented in Section 6, where the implementation is verified with the method of manufactured solutions. Additionally, results from steady-state computations are compared with the predicted convergence rates from linear theory. Finally conclusions are drawn in Section 7. 


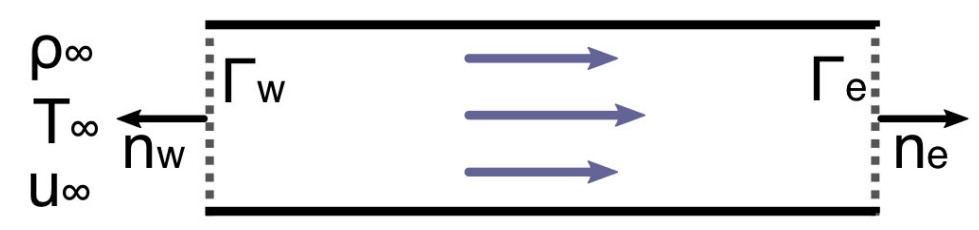

Figure 1: Illustration of the domain. The north and south boundaries are solid walls and the east $\left(\Gamma_{e}\right)$ and west $\left(\Gamma_{w}\right)$ are artificial.

\section{Problem description and the governing equations}

Consider the Euler equations on a rectangular domain as exemplified in Figure 1. The north and south boundaries of the domain are solid walls, i.e. the normal velocity is zero. The west and east boundaries are artificial, where the investigated boundary conditions are imposed. The flow upstream and downstream of the computational domain is assumed constant and denoted by the subscript $\infty$. We assume that the changes in the flow are small and gradual, which makes the flow isentropic [15] and amenable for linearization.

In a non-dimensional form, the governing nonlinear Euler equations are

$$
\vec{u}_{t}+F(\vec{u})_{x}+G(\vec{u})_{y}=0
$$

where $\vec{u}=(\rho, \rho u, \rho v, e)^{\top}$ and the fluxes are

$F(\vec{u})=\left(\rho u, \rho u^{2}+p, \rho u v,(e+p) u\right)^{\top} \quad$ and $\quad G(\vec{u})=\left(\rho v, \rho u v, \rho v^{2}+p,(e+p) v\right)^{\top}$.

The quantities $\rho, u, v, p$ are the density, the velocities in the $x, y$-direction, the pressure and $e=p /(\gamma-1)+\rho\left(u^{2}+v^{2}\right) / 2$ the total energy, respectively. We have used the subscripts $t, x, y$ to denote the partial derivatives with respect to time and space. Furthermore, the perfect gas law takes the form $M_{\infty}^{2} \gamma p=\rho T$, where $T$ is the temperature and $\gamma$ is the heat capacity ratio. We have introduced the free-stream Mach number $M_{\infty}=U_{\infty} / c_{\infty}$, where $U_{\infty}$ and $c_{\infty}$ are the free-stream speed and speed of sound, respectively. The local speed of sound is $c^{2}=T / M_{\infty}^{2}$. The first two boundary conditions specified at the inflow (assuming isentropic flow) are

$$
\begin{array}{ll}
P_{0}=p\left[1+\left(u^{2}+v^{2}\right)(\gamma-1) M_{\infty}^{2} /(2 T)\right]^{\gamma /(\gamma-1)} & =g_{1}, \\
T_{0}=T+\left(u^{2}+v^{2}\right)(\gamma-1) M_{\infty}^{2} / 2 & =g_{2},
\end{array}
$$

which are the total pressure and temperature, respectively. 
Remark 1. Preceding the upcoming analysis we stress that boundary conditions of the general form $L u=g$ must have $i$ ) an appropriate boundary operator $(L)$ for boundedness and $i i)$ available data $(g)$ for accuracy. Without these two requirements, meaningful calculations are not possible.

\section{The continuous linearized problem}

The energy method require symmetric matrices [16], which can be obtained from the linearized frozen-coefficient representation of (1). We make the change of variables $(\rho, \rho u, \rho v, e)^{\top} \rightarrow(\rho, u, v, p)^{\top}[17]$ and linearize around a constant free-stream flow [13]. Both the north and the south boundaries are solid walls (see Figure 1), and therefore $\bar{v}=0$. The constant state (indicated by an overbar) we consider is

$$
\bar{\rho}=\bar{u}=\bar{T}=1, \quad \bar{v}=0, \quad \bar{c}=1 / M_{\infty} .
$$

The constant state (3) is used in all upcoming computations unless otherwise explicitly specified. Further, for dry air (the typical working fluid for gas turbines) $\gamma=1.4$, which leaves $M_{\infty}$ as the only parameter. We postulate that the direction of the main flow is in the positive $x$-direction and that the speed is subsonic, $0<\bar{u}<\bar{c}$, since $M_{\infty}<1$.

By using the symmetrizers from [18], the linear symmetric system becomes

$$
W_{t}+A W_{x}+B W_{y}=0
$$

where $W=\left[\rho \bar{c} /(\sqrt{\gamma} \bar{\rho}), u, v, T /\left(\bar{c} M_{\infty}^{2} \sqrt{\gamma(\gamma-1)}\right)\right]^{\top}$. The quantities $\rho, u, v, T$ are small perturbations from the constant state and the symmetrized matrices are

$$
\begin{aligned}
A & =\left(\begin{array}{cccc}
\bar{u} & \bar{c} / \sqrt{\gamma} & 0 & 0 \\
\bar{c} / \sqrt{\gamma} & \bar{u} & 0 & \bar{c} \sqrt{(\gamma-1) / \gamma} \\
0 & 0 & \bar{u} & 0 \\
0 & \bar{c} \sqrt{(\gamma-1) / \gamma} & 0 & \bar{u}
\end{array}\right), \\
B & =\left(\begin{array}{cccc}
0 & 0 & \bar{c} / \sqrt{\gamma} & 0 \\
0 & 0 & 0 & 0 \\
\bar{c} / \sqrt{\gamma} & 0 & 0 & \bar{c} \sqrt{(\gamma-1) / \gamma} \\
0 & 0 & \bar{c} \sqrt{(\gamma-1) / \gamma} & 0
\end{array}\right) .
\end{aligned}
$$

In the upcoming analysis, all boundaries except the inflow boundary $\Gamma_{w}$ with its outward-pointing unit normal $n_{w}=(-1,0)$ will be disregarded redand are assumed to be treated in a correct manner. 


\subsection{The energy method and well-posedness}

The IBVP that we will analyze is (4) augmented with initial and boundary conditions

$$
\begin{array}{rlrl}
W_{t}+A W_{x}+B W_{y}=0, & (x, y) \in \Omega, & t \geq 0 \\
L W=g, & (x, y) \in \partial \Omega, & t \geq 0 \\
W=f, & (x, y) \in \Omega, \quad t=0 .
\end{array}
$$

In $(5), \Omega=[0,1]^{2}, \partial \Omega$ denotes the boundary to $\Omega, L$ is the boundary operator and $f$ and $g$ are smooth functions that are considered to be known. We introduce inner products and the corresponding norm

$$
\langle U, V\rangle_{\Omega}=\iint_{\Omega} U^{\top} V d \Omega, \quad\langle U, V\rangle_{\Gamma}=\int_{\Gamma} U^{\top} V d S, \quad\|U\|^{2}=\langle U, U\rangle_{\Omega},
$$

where $U, V \in \mathcal{L}^{2}(\Omega)$ and $\Gamma \subset \partial \Omega$.

Definition 1. The IBVP (5) with $g=0$ is well posed if a unique solution exists satisfying

$$
\|W(\cdot, t)\|^{2} \leq K_{c} e^{\alpha_{c} t}\|f(\cdot)\|^{2},
$$

where $K_{c}$ and $\alpha_{c}$ are constants.

Applying the energy method to (5) results in

$$
\|W\|_{t}^{2}-\langle W, A W\rangle_{\Gamma_{w}}=0,
$$

where all boundary terms except the west one's have been neglected. Since $A$ is symmetric, it can be decomposed as $A=X \Lambda X^{\top}$, where

$$
\begin{aligned}
X & =\left(\begin{array}{cccc}
1 / \sqrt{2 \gamma} & 1 / \sqrt{2 \gamma} & -\sqrt{(\gamma-1) / \gamma} & 0 \\
1 / \sqrt{2} & -1 / \sqrt{2} & 0 & 0 \\
0 & 0 & 0 & 1 \\
\sqrt{(\gamma-1) /(2 \gamma)} & \sqrt{(\gamma-1) /(2 \gamma)} & 1 / \sqrt{\gamma} & 0
\end{array}\right), \\
\Lambda & =\operatorname{diag}(\bar{u}+\bar{c}, \bar{u}-\bar{c}, \bar{u}, \bar{u}) .
\end{aligned}
$$

The corresponding characteristic variables become $q=\left(q_{1}, q_{2}, q_{3}, q_{4}\right)^{\top}=$ $X^{\top} W$. Let $\Lambda^{+}=\operatorname{diag}(\bar{u}+\bar{c}, \bar{u}, \bar{u})$ and $\Lambda^{-}=\bar{u}-\bar{c}$ denote the positive and negative eigenvalues respectively, then (6) takes the form

$$
\|W\|_{t}^{2}-\left\langle\left(\begin{array}{c}
q^{+} \\
q^{-}
\end{array}\right),\left(\begin{array}{cc}
\Lambda^{+} & 0 \\
0 & \Lambda^{-}
\end{array}\right)\left(\begin{array}{l}
q^{+} \\
q^{-}
\end{array}\right)\right\rangle_{\Gamma_{w}}=0,
$$


where $q^{+}=\left(q_{1}, q_{3}, q_{4}\right)^{\top}$ and $q^{-}=q_{2}$.

Following [7], the boundary conditions are written in a general form where the ingoing characteristics are specified in terms of the outgoing ones and data as

$$
q^{+}-R q^{-}=g
$$

where $R \in \mathbb{R}^{3 \times 1}$. Imposing the homogeneous $(g=0)$ form of (8) yields $\|W\|_{t}^{2}-\left\langle q^{-},\left(\Lambda^{-}+R^{\top} \Lambda^{+} R\right) q^{-}\right\rangle_{\Gamma_{w}}=0$. Integrating in time, we find that

$$
\|W(t)\|^{2}-\int_{0}^{t}\left\langle q^{-},\left(\Lambda^{-}+R^{\top} \Lambda^{+} R\right) q^{-}\right\rangle_{\Gamma_{w}} d \tau=\|f\|^{2} .
$$

A suitable energy estimate is hence obtained if $\Lambda^{-}+R^{\top} \Lambda^{+} R$ is non-positive. The energy estimate leads directly to uniqueness and existence is given by the fact that we use a minimal number of boundary conditions. The minimal number of boundary conditions is equal to the number of nonzero entries in $\Lambda^{+}$. We have proven

Proposition 1. The continuous problem (5) with homogeneous boundary conditions on the form (8) is well posed if

$$
\Lambda^{-}+R^{\top} \Lambda^{+} R \leq 0
$$

Remark 2. The result and proof of Proposition 1 are given in [7] and included here for clarification and completeness. In [7], a strong estimate for the non-homogeneous form of (8) is also given.

\subsection{The boundary operator}

We linearize the nonlinear boundary conditions (2) to obtain

$$
\begin{aligned}
\rho \bar{u}^{2} / 2+u \bar{\rho} \bar{u}+p\left[1-\bar{u}^{2} /\left(2 \bar{c}^{2}\right)\right] & =g_{1}, \\
-\rho \gamma \bar{p} / \bar{\rho}^{2}+u \bar{u}(\gamma-1)+p \gamma / \bar{\rho} & =g_{2} .
\end{aligned}
$$

The third boundary condition (we need three since $\Lambda^{+}$has three nonzero entries for subsonic flow) that we consider is given by

$$
\sigma_{1} \bar{c}^{2} \rho+\sigma_{2} \bar{\rho} \bar{c} u+\sigma_{3} \bar{\rho} \bar{c} v+\sigma_{4} p=g_{3}
$$


which is a linear combination of the state variables with dimensionless weights $\sigma_{1}, \sigma_{2}, \sigma_{3}, \sigma_{4}$. Our ambition is to choose the weights $\sigma_{i}, i=1, \ldots, 4$ such that an energy estimate is obtained. We combine (11) and (12) to obtain the boundary operator in (5) as

$$
\underbrace{\left(\begin{array}{cccc}
\frac{\bar{\rho}\left[2 \bar{c}^{2}+(\gamma-1) \bar{u}^{2}\right]}{2 \bar{c} \sqrt{\gamma}} & \bar{\rho} \bar{u} & 0 & \bar{\rho} \bar{c}\left(1-\frac{\bar{u}^{2}}{\left(2 \bar{c}^{2}\right)}\right) \sqrt{\frac{(\gamma-1)}{\gamma}} \\
0 & (\gamma-1) \bar{u} & 0 & \bar{c} \sqrt{(\gamma-1) \gamma} \\
\frac{\bar{\rho} \bar{c}\left(\gamma \sigma_{1}+\sigma_{4}\right)}{\sqrt{\gamma}} & \sigma_{2} \bar{\rho} \bar{c} & \sigma_{3} \bar{\rho} \bar{c} & \bar{\rho} \bar{c} \sigma_{4} \sqrt{\frac{(\gamma-1)}{\gamma}}
\end{array}\right)}_{L} W=\left(\begin{array}{l}
g_{1} \\
g_{2} \\
g_{3}
\end{array}\right) .
$$

Provided that $\sigma_{3} \neq 0$, the boundary matrix in (8) can be obtained by the transformations

$$
L W=g \Longleftrightarrow\left(L_{X}^{+}\right)^{-1}\left(L_{X}^{+} q^{+}+L_{X}^{-} q^{-}\right)=\left(L_{X}^{+}\right)^{-1} g \Longleftrightarrow q^{+}-R q^{-}=\left(L_{X}^{+}\right)^{-1} g .
$$

The matrices $L_{X}^{+}$and $L_{X}^{-}$are the columns in $L X$ that correspond to $q^{+}$and $q^{-}$respectively and

$$
R=-\left(L_{X}^{+}\right)^{-1} L_{X}^{-}=\left(\begin{array}{c}
(\bar{u}-\bar{c}) /(\bar{u}+\bar{c}) \\
0 \\
\sqrt{2}\left[\bar{c} \sigma_{2}-\bar{u}\left(\sigma_{1}+\sigma_{4}\right)\right] /\left(\sigma_{3}(\bar{u}+\bar{c})\right)
\end{array}\right) .
$$

Condition (10) now becomes

$$
\Lambda^{-}+R^{\top} \Lambda^{+} R=f\left(\sigma_{1}, \sigma_{2}, \sigma_{3}, \sigma_{4}\right) \leq 0
$$

where

$$
f=\frac{2\left[\left(\sigma_{1}+\sigma_{4}\right)^{2} \bar{u}^{3}+\sigma_{2}^{2} \bar{u} \bar{c}^{2}+\sigma_{3}^{2} \bar{u}\left(\bar{u}^{2}-\bar{c}^{2}\right)-2 \sigma_{2}\left(\sigma_{1}+\sigma_{4}\right) \bar{c} \bar{u}^{2}\right]}{\left[\sigma_{3}(\bar{u}+\bar{c})\right]^{2}} .
$$

We aim for weights $\sigma_{i}, i=1, \ldots 4$ such that the inequality in (14) holds. Let $(\nabla f)_{i}=\partial f / \partial \sigma_{i}, i=1 \ldots 4$ denote the gradient of $f$. Stationary points to $f$ are given by

$$
\nabla f=\frac{4}{\sigma_{3}^{2}(\bar{u}+\bar{c})^{2}}\left(\begin{array}{c}
\left(\sigma_{1}+\sigma_{4}\right) \bar{u}^{3}-\sigma_{2} \bar{c} \bar{u}^{2} \\
\sigma_{2} \bar{u} \bar{c}^{2}-\left(\sigma_{1}+\sigma_{4}\right) \bar{c} \bar{u}^{2} \\
\frac{\left.2 \sigma_{2}\left(\sigma_{1}+\sigma_{4}\right) \bar{c} \bar{u}^{2}-\left(\sigma_{1}+\sigma_{4}\right)^{2} \bar{u}^{3}-\sigma_{2}^{2} \bar{u}^{2}\right]}{\sigma_{3}} \\
\left(\sigma_{1}+\sigma_{4}\right) \bar{u}^{3}-\sigma_{2} \bar{c} \bar{u}^{2}
\end{array}\right)=0
$$


which yields $\sigma_{2}=\left(\sigma_{1}+\sigma_{4}\right)(\bar{u} / \bar{c})$. To classify the stationary points, we examine the difference $\delta f=f\left[\sigma_{1}+i,\left(\sigma_{1}+\sigma_{4}\right) \bar{u} / \bar{c}+j, \sigma_{3}+k, \sigma_{4}+l\right]-$ $f\left[\sigma_{1},\left(\sigma_{1}+\sigma_{4}\right) \bar{u} / \bar{c}, \sigma_{3}, \sigma_{4}\right]$. If $\delta f$ is non-negative for all $i, j, k, l$ sufficiently small, then the stationary points are all local minima. We get

$$
\delta f=2 \bar{u}[\bar{c} j+(i+l) \bar{u}]^{2} /\left[\left(\sigma_{3}+k\right)(\bar{u}+\bar{c})\right]^{2} \geq 0,
$$

which means that all stationary points are minima. One convenient choice is therefore $\sigma_{1}=\sigma_{4}=\sigma_{2}=0$ and $\sigma_{3}=1 /(\bar{\rho} \bar{c})$, which turns (12) into

$$
v=0 \text {. }
$$

Hence, the final set of inflow conditions that we will consider is:

$$
\begin{aligned}
\rho \bar{u}^{2} / 2+u \bar{\rho} \bar{u}+p\left[1-\bar{u}^{2} /\left(2 \bar{c}^{2}\right)\right] & =g_{1}, \\
-\rho \gamma \bar{p} / \bar{\rho}^{2}+u \bar{u}(\gamma-1)+p \gamma / \bar{\rho} & =g_{2}, \\
v & =0 .
\end{aligned}
$$

With (17), we find $R=[(\bar{u}-\bar{c}) /(\bar{u}+\bar{c}), 0,0]^{\top}$ and condition (14) becomes

$$
\Lambda^{-}+R^{\top} \Lambda^{+} R=2 \bar{u}(\bar{u}-\bar{c}) /(\bar{u}+\bar{c})<0 .
$$

Thus, the three inflow conditions (17) forms a well-posed boundary formulation.

Remark 3. Note that (17) corresponds to a set of inflow conditions for the nonlinear problem for which the boundary data are physically measurable and hence possible to use in practice.

\section{The spectrum}

A solution to a hyperbolic problem may vanish at the boundaries but remain non-zero in the interior of the domain. Hence, the energy method does not provide sufficient information about the convergence rate to steady state. A necessary condition is that the solution vanishes as $t \rightarrow \infty$ for any initial data $f$ when $g=0$ in (5). We use the Laplace transform method [10] in order to investigate if that is the case. 
Consider the linear system (5) with homogeneous inflow and outflow conditions:

$$
\begin{aligned}
W_{t}+A W_{x}+B W_{y} & =0, & & (x, y) \in \Omega, & & t \geq 0, \\
L_{0} W & =0, & & (x, y) \in \Gamma_{w}, & & t \geq 0, \\
L_{1} W & =0, & & (x, y) \in \Gamma_{e}, & & t \geq 0, \\
W & =f, & & (x, y) \in \Omega, & & t=0,
\end{aligned}
$$

posed on the domain $\Omega=[0,1] \times[-\pi, \pi]$. The inflow and outflow boundaries are denoted $\Gamma_{w}=\{(x, y) \in \Omega: x=0\}$ and $\Gamma_{e}=\{(x, y) \in \Omega: x=1\}$, respectively. The inflow conditions (17) are imposed by $L_{0}$, while $L_{1}$ imposes the static pressure at the outflow boundary (a well-posed subsonic outflow condition [19]).

We consider the solution to be $2 \pi$-periodic in the y-direction, such that a Fourier expansion can be used, and apply the Laplace transform in time:

$$
W(x, y, t)=\sum_{\omega=-\infty}^{\infty} \tilde{W}(x, \omega, t) e^{i \omega y}, \quad \hat{W}(x, \omega, s)=\int_{0}^{\infty} e^{-s t} \tilde{W}(x, \omega, t) d t .
$$

In (19), $s=\eta+i \xi$ and $\eta, \xi \in \mathbb{R}$. All integrals exist since only smooth and bounded solutions are considered. Applying (19) to (18) yields for every $\omega$

$$
\begin{aligned}
(s I+i \omega B) \hat{W}+A \hat{W}_{x} & =\tilde{f}(x, \omega), & & x \in[0,1], \\
L_{0} \hat{W} & =0, & & x=0, \\
L_{1} \hat{W} & =0, & & x=1,
\end{aligned}
$$

where $\tilde{f}(x, \omega)$ are the Fourier coefficients of $f$ and $I$ is the identity matrix. The solution $\hat{W}=\hat{W}_{h}+\hat{W}_{p}$ to (20) consists of a homogeneous and a particular solution. The particular solution depends on the initial data and can be considered known.

By making the ansatz $\hat{W}_{h}=\psi e^{\kappa x}$, where $\psi \in \mathbb{C}^{4 \times 1}$ and $\kappa \in \mathbb{C}$, we find that

$$
(s I+i \omega B+\kappa A) \psi=0
$$

holds. For non-trivial solutions,

$$
\operatorname{det}(s I+i \omega B+\kappa A)=(s+\kappa \bar{u})^{2}\left[\left(s+\kappa \bar{u}^{2}\right)^{2}+\bar{c}^{2}\left(\omega^{2}-\kappa^{2}\right)\right]=0
$$

must be satisfied, where $\operatorname{det}(\cdot)$ denotes the determinant. The roots to $(22)$ with respect to $\kappa$ are

$$
\kappa_{1,2}=(\bar{u} s \pm \bar{c} \alpha) /\left(\bar{c}^{2}-\bar{u}^{2}\right), \quad \kappa_{3}=\kappa_{4}=-s / \bar{u},
$$


where $\alpha=\sqrt{s^{2}+\omega^{2}\left(\bar{c}^{2}-\bar{u}^{2}\right)}$. The two distinct roots $\kappa_{1}$ and $\kappa_{2}$ inserted in (21) yield

$$
\begin{aligned}
& \psi_{1}=\left(1, \kappa_{1} \beta_{+}, i \omega\left(\bar{c}^{2}-\bar{u}^{2}\right) \beta_{+}, \sqrt{\gamma-1}\right)^{\top}, \\
& \psi_{2}=\left(1, \kappa_{2} \beta_{-}, i \omega\left(\bar{c}^{2}-\bar{u}^{2}\right) \beta_{-}, \sqrt{\gamma-1}\right)^{\top},
\end{aligned}
$$

where $\beta_{ \pm}=-\sqrt{\gamma}[\bar{c} s \pm \bar{u} \alpha] /\left[\bar{c}^{2} s^{2}+\bar{u}^{2} \alpha^{2} \pm 2 s \bar{c} \alpha\right]$. For the repeated root $\kappa_{3}=\kappa_{4}=-s / \bar{u}$, we reformulate the ansatz to $\hat{W}_{h}=(\psi+x \phi) e^{\kappa_{3} x}$, where $\phi \in \mathbb{C}^{4 \times 1}$, and get the system of linearly independent equations

$$
\begin{aligned}
\left(s I+i \omega B+\kappa_{3} A\right) \phi & =0, \\
\left(s I+i \omega B+\kappa_{3} A\right) \psi+A \phi & =0 .
\end{aligned}
$$

We first solve (23a) to obtain $\phi$, which is inserted into (23b). The solution to the whole system (23) becomes $\phi=(0,0,0,0)^{\top}$ and $\psi=\sigma_{3} \psi_{3}+\sigma_{4} \psi_{4}$, where $\psi_{3}=(-\sqrt{\gamma-1}, 0,0,1)^{\top}$ and $\psi_{4}=(0, i \omega \bar{u}, s, 0)^{\top}$. Finally, the homogeneous solution to $(20)$ is $\hat{W}_{h}=\Psi K(x) \vec{\sigma}$, where $\Psi=\left[\psi_{1}, \psi_{2}, \psi_{3}, \psi_{4}\right]$, $K(x)=\operatorname{diag}\left(e^{\kappa_{1}}, e^{\kappa_{2}}, e^{\kappa_{3}}, e^{\kappa_{3}}\right)$ and $\vec{\sigma}=\left(\sigma_{1}, \sigma_{2}, \sigma_{3}, \sigma_{4}\right)^{\top}$ contain the solution vectors, the exponential functions and the parameters that will be determined from the boundary conditions, respectively.

Inserting $\hat{W}$ into the boundary conditions yields for every $\omega$ and $M_{\infty}$

$$
E(s) \vec{\sigma}=\left(\begin{array}{l}
L_{0} \Psi K(0) \\
L_{1} \Psi K(1)
\end{array}\right) \vec{\sigma}=H
$$

In $(24), E(s) \in \mathbb{C}^{4 \times 4}$ (three inflow conditions and one outflow condition) and $H$ depends on the particular solution. The spectrum to the problem is given by $s^{*}=\{s \in \mathbb{C}: \operatorname{det}(E(s))=0\}$. We have a well-posed problem if all $s^{*}$ are located in the left half of the complex plane [10]. Let $\eta_{C}^{*}=\max \mathscr{R}\left(s^{*}\right)$ denote the real part of the eigenvalues closest to the imaginary axis. If $\mathscr{R}(s)>\eta_{C}^{*}$, we can solve for $\vec{\sigma}$ and formally transform back to the time domain by taking the inverse Laplace transform:

$$
\tilde{W}(x, \omega, t)=e^{\eta_{C}^{*} t} \frac{1}{2 \pi i} \int_{-i \infty}^{i \infty} \hat{W}\left(x, \omega, \eta_{C}^{*}+i \xi\right) e^{i \xi t} d \xi
$$

The solution will converge to steady state independent of the initial condition if $\eta_{C}^{*}<0$.

The singularities to (24) are found with the Secant method. Initial values on a grid are used such that all roots within a certain domain around the 


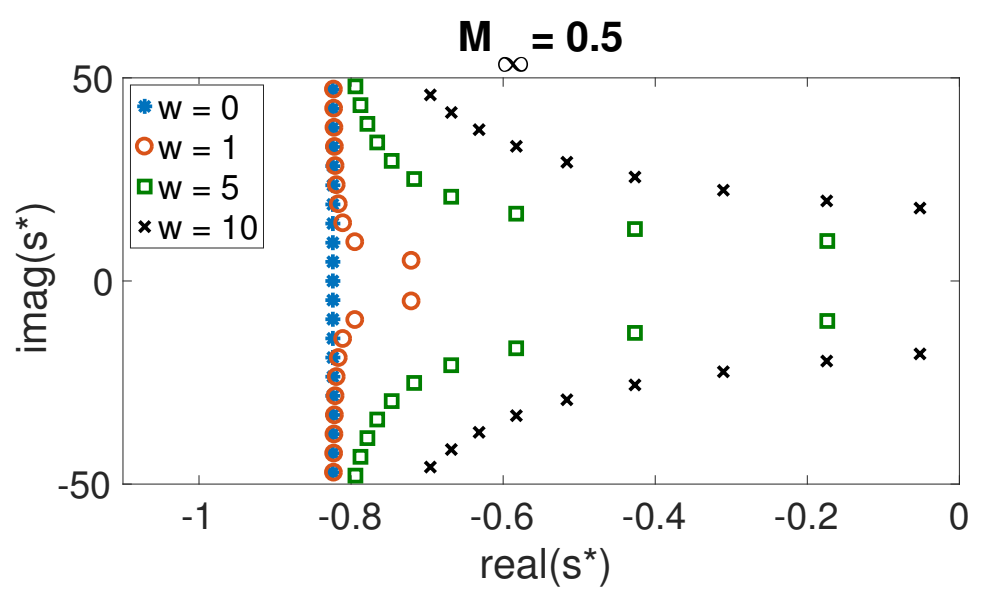

Figure 2: The continuous spectrum for $M_{\infty}=0.5$ and different $\omega$.

\begin{tabular}{c|c|c|c}
$w$ & $M_{\infty}=0.1$ & $M_{\infty}=0.5$ & $M_{\infty}=0.9$ \\
\hline 0 & -0.9933 & -0.8240 & -0.3108 \\
1 & -0.9009 & -0.7206 & -0.1558 \\
5 & -0.2784 & -0.1737 & -0.0134 \\
10 & -0.0881 & -0.0518 & -0.0031 \\
\hline
\end{tabular}

Table 1: The largest real part of the spectrum $\left(\eta_{C}^{*}\right)$ for different $M_{\infty}$ and $\omega$.

origin are found. Spectra for $M_{\infty}=0.5$ and different $\omega$ are illustrated in Figure 2. Similar results are obtained for $M_{\infty}=0.1$ and $M_{\infty}=0.9$. All spectra resides in the left half of the complex plane. As $|\omega|$ increases, the spectrum moves closer to the imaginary axis, but stays in the left part of the complex plane, a behavior also observed in [20]. Moreover, the spectrum also moves closer to the imaginary axis as $M_{\infty}$ increases, but stays in the left half of the complex plane for $M_{\infty}<1$. Table 1 presents the $\eta_{C}^{*}$ extracted from the spectra. As can be seen from the table, all cases converge to steady state.

\section{The semi-discrete scheme}

Let the domain $\Omega=[0,1]^{2}$ be discretized with $N+1$ and $M+1$ grid points; $x_{i}=i / N, i=0, \ldots, N$ and $y_{j}=j / M, j=0, \ldots, M$. Further, let the solution field be $\mathbf{W}^{i j k}(t) \approx W\left(x_{i}, y_{j}, k, t\right)$, where $i, j$ correspond to the grid point and $k$ to the corresponding dependent variable. 
The SBP approximation of the spatial derivatives are given by
$\mathbf{D}_{x} \mathbf{W}=\left(P_{x}^{-1} Q_{x} \otimes I_{y} \otimes I_{4}\right) \mathbf{W} \approx \vec{W}_{x}$,
$\mathbf{D}_{y} \mathbf{W}=\left(I_{x} \otimes P_{y}^{-1} Q_{y} \otimes I_{4}\right) \mathbf{W} \approx \vec{W}_{y}$,

where $\otimes$ denotes the Kronecker product and $\vec{W}_{x, y}$ contain the partial derivatives evaluated on the grid. The matrices $P_{x, y}$ are diagonal and positive definite, such that $\mathbf{P}=P_{x} \otimes P_{y} \otimes I_{4}$ forms a quadrature rule that defines the norm $\|\mathbf{W}\|_{\mathbf{P}}^{2}=\mathbf{W}^{\top} \mathbf{P} \mathbf{W} \approx \iint_{\Omega} W^{\top} W d \Omega$. Furthermore, the matrices $Q_{x, y}$ satisfy the SBP-property

$$
Q_{x}+Q_{x}^{\top}=E_{N}-E_{0}, \quad Q_{y}+Q_{y}^{\top}=E_{M}-E_{0},
$$

where $E_{0}=\operatorname{diag}(1,0,0, \ldots, 0)$ and $E_{N, M}=\operatorname{diag}(0,0,0, \ldots, 1)$ are matrices of appropriate sizes.

We will also need the matrices

$$
\mathbf{A}=I_{x} \otimes I_{y} \otimes A, \quad \mathbf{B}=I_{x} \otimes I_{y} \otimes B, \quad \mathbf{X}=I_{x} \otimes I_{y} \otimes X, \quad \boldsymbol{\Lambda}=I_{x} \otimes I_{y} \otimes \Lambda,
$$

where $\mathbf{A}=\mathbf{X} \boldsymbol{\Lambda} \mathbf{X}^{\top}$. Let $\boldsymbol{\Lambda}^{ \pm}=(\boldsymbol{\Lambda} \pm|\boldsymbol{\Lambda}|) / 2$ and $\mathbf{X}^{ \pm}=I_{x} \otimes I_{y} \otimes X^{ \pm}$, where

$$
\begin{aligned}
X^{+} & =\left(\begin{array}{cccc}
1 / \sqrt{2 \gamma} & 0 & -\sqrt{(\gamma-1) / \gamma} & 0 \\
1 / \sqrt{2} & 0 & 0 & 0 \\
0 & 0 & 0 & 1 \\
\sqrt{(\gamma-1) /(2 \gamma)} & 0 & 1 / \sqrt{\gamma} & 0
\end{array}\right), \\
X^{-} & =\left(\begin{array}{cccc}
0 & 1 /(\sqrt{2 \gamma}) & 0 & 0 \\
0 & -1 / \sqrt{2} & 0 & 0 \\
0 & 0 & 0 & 0 \\
0 & \sqrt{(\gamma-1) /(2 \gamma)} & 0 & 0
\end{array}\right)
\end{aligned}
$$

The characteristic variables in semi-discrete form are $\mathbf{q}=\mathbf{X}^{\top} \mathbf{W}$ and $\mathbf{q}^{ \pm}=$ $\left(\mathbf{X}^{ \pm}\right)^{\top} \mathbf{W}$.

Finally, the semi-discrete formulation of (5) becomes

$$
\begin{array}{rlrl}
\mathbf{W}_{t}+\left(\mathbf{D}_{x} \mathbf{A}+\mathbf{D}_{y} \mathbf{B}\right) \mathbf{W} & =\mathbf{P}^{-1} \mathbf{P}_{y}^{0} \boldsymbol{\Sigma}\left(\mathbf{q}^{+}-\mathbf{R q}^{-}-\overline{\mathbf{g}}\right)+\text { Pen, }, & t \geq 0 \\
\mathbf{W} & =\mathbf{f} & t & =0 .
\end{array}
$$

All penalty terms except the west one's are included in the term Pen and will not be discussed further. We have introduced $\mathbf{P}_{y}^{0}=E_{0} \otimes P_{y} \otimes I_{4}$ and $\mathbf{f}$, which is the quadrature formula over the west boundary and the initial data 
evaluated on the grid, respectively. The inflow conditions (17) are imposed weakly on the form (8) and $\mathbf{R}=I_{x} \otimes I_{y} \otimes R$, where $R$ is a matrix corresponding to the continuous boundary conditions. Furthermore, $\Sigma$ is a penalty matrix that will be determined for stability. We make the following definition

Definition 2. The semi-discrete scheme (25) with $\mathbf{g}=\mathbf{0}$ is stable if

$$
\|\mathbf{W}(t)\|_{\mathbf{P}}^{2} \leq K_{d} e^{\alpha_{d} t}\|\mathbf{f}\|_{\mathbf{P}}^{2}
$$

where $K_{d}, \alpha_{d}$ are constants.

Following the procedure outlined in [7], we choose $\boldsymbol{\Sigma}=-\mathbf{X}^{+} \boldsymbol{\Lambda}^{+}$and apply the discrete energy method to $(25)$ with $\mathbf{g}=\mathbf{0}$ to obtain the estimate

$$
\begin{aligned}
& \|\mathbf{W}(t)\|_{\mathbf{P}}^{2}-\int_{0}^{t}\left(\mathbf{q}^{-}\right)^{\top} \mathbf{P}_{y}^{0}\left(\Lambda^{-}+R^{\top} \Lambda^{+} R\right) \mathbf{q}^{-} d \tau= \\
& \|\mathbf{f}\|_{\mathbf{P}}^{2}-\int_{0}^{t}\left(\mathbf{q}^{+}-\mathbf{R} \mathbf{q}^{-}\right)^{\top} \mathbf{P}_{y}^{0} \boldsymbol{\Lambda}^{+}\left(\mathbf{q}^{+}-\mathbf{R} \mathbf{q}^{-}\right) d \tau
\end{aligned}
$$

Since Proposition 1 holds, $\left(\Lambda^{-}+R^{\top} \Lambda^{+} R\right) \leq 0$, and we have proved

Proposition 2. The scheme (25) with $\boldsymbol{\Sigma}=-\mathbf{X}^{+} \boldsymbol{\Lambda}^{+}$is stable.

The estimate in (26) mimics the continuous estimate (9) and the last term in (26) adds dissipation to the scheme.

The provably stable linear scheme (25) must be related to the nonlinear scheme. Let the solution field $\overrightarrow{\mathbf{u}}(t)$ hold the conservative variables, ordered in the same way as $\mathbf{W}(t)$ in (25). Further, let $\mathbf{M}$ be the transformation matrix between the conservative and symmetric variables such that $\overrightarrow{\mathbf{u}}=\mathbf{M W}$ and $\mathbf{W}=\mathbf{M}^{-1} \overrightarrow{\mathbf{u}}$. Multiplying (25) by $\mathbf{M}$ yields

$$
\begin{array}{rlrl}
\overrightarrow{\mathbf{u}}_{t}+\mathbf{D}_{x} \overrightarrow{\mathbf{F}}+\mathbf{D}_{y} \overrightarrow{\mathbf{G}} & =\mathbf{P}^{-1} \mathbf{P}_{y}^{0} \overline{\mathbf{\Sigma}}\left(\mathbf{L}_{\mathbf{x}}^{+}\right)^{-1}(\mathbf{L} \overrightarrow{\mathbf{u}}-\mathbf{g})+\text { MPen } & t \geq 0 \\
\overrightarrow{\mathbf{u}} & =\mathbf{M f} & & t=0
\end{array}
$$

where $\overrightarrow{\mathbf{F}}$ and $\overrightarrow{\mathbf{G}}$ are the flux vectors. The penalty matrix is $\overline{\boldsymbol{\Sigma}}=\mathbf{M} \boldsymbol{\Sigma}$, where $\boldsymbol{\Sigma}=-\mathbf{X}^{+} \boldsymbol{\Lambda}^{+}$as in (25). More details are found in the Appendix. 


\subsection{The discrete spectrum}

Let $\Omega^{\prime}=[0,1]$ be discretized with $N+1$ grid points; $x_{i}=i / N, i=0, \ldots, N$ and consider the discrete scheme

$$
\begin{aligned}
\mathbf{W}_{t}+\mathbf{M W} & =\mathbf{0}, & t \geq 0, \\
\mathbf{W} & =\mathbf{f}, & t=0,
\end{aligned}
$$

where $\mathbf{M}=D_{x} \otimes A+i \omega\left(I_{x} \otimes B\right)-P_{x}^{-1} E_{0} \otimes X^{+} \Lambda^{+} H_{0}+P_{x}^{-1} E_{N} \otimes X^{-} \Lambda^{-} H_{1}$ and where $D_{x}$ is an SBP operator [14]. Further, $H_{0}$ imposes (17) at the inflow boundary and $H_{1}$ the pressure at the outflow boundary. By applying the Laplace transform in time to (28), we obtain

$$
\left(s\left(I_{x} \otimes I_{4}\right)+\mathbf{M}\right) \hat{\mathbf{W}}=\mathbf{f},
$$

which is the discrete formulation of (20). As in the continuous case, the solution $\hat{\mathbf{W}}=\hat{\mathbf{W}}_{h}+\hat{\mathbf{W}}_{p}$ consists of a homogeneous and particular solution, where the particular solution depends on the initial data and can be considered known. Only the homogeneous part is of interest and (29) turns into the eigenvalue problem $\left(s\left(I_{x} \otimes I_{4}\right)+\mathbf{M}\right) \hat{\mathbf{W}}=0$. The discrete spectrum is defined as $\left\{s \in \mathbb{C}: \operatorname{det}\left(s\left(I_{x} \otimes I_{4}\right)+\mathbf{M}\right)=0\right\}$ and it is obtained by computing the eigenvalues to $\mathbf{M}$.

Figure 3-6 illustrates the discrete spectra for different number of grid points for the 4th order SBP approximation [14] when $M_{\infty}=0.5$ and $\omega$ is varied. The continuous spectra is included and, as can be seen in the figures, the discrete spectra converge to its continuous analogue as the number of grid points is increased. All spectra resides in the left half of the complex plane. Similar results are obtained for $M_{\infty}=0.1$ and $M_{\infty}=0.9$. In the

continuous case, the singularity with the largest real part $\left(\eta_{C}^{*}\right)$ determines the decay rate. For the discrete scheme, it is similarly the eigenvalue to $\mathbf{M}$ with largest real part (denoted $\eta_{D}^{*}$ ) that determines the decay rate. From Figure $3-6$, we can see that $\eta_{C}^{*}$ is well approximated by $\eta_{D}^{*}$ for all cases.

\section{Nonlinear Computations}

The linear equations (5) and the corresponding discrete approximation (25) serve as analytical tools, while it is the nonlinear equations (1) that must be used to obtain realistic results. A nonlinear compressible Euler solver based on the SBP-SAT technique is used for the following computations on the domain $\Omega=[0,1]^{2}$. The north and south boundaries are (as in Figure 


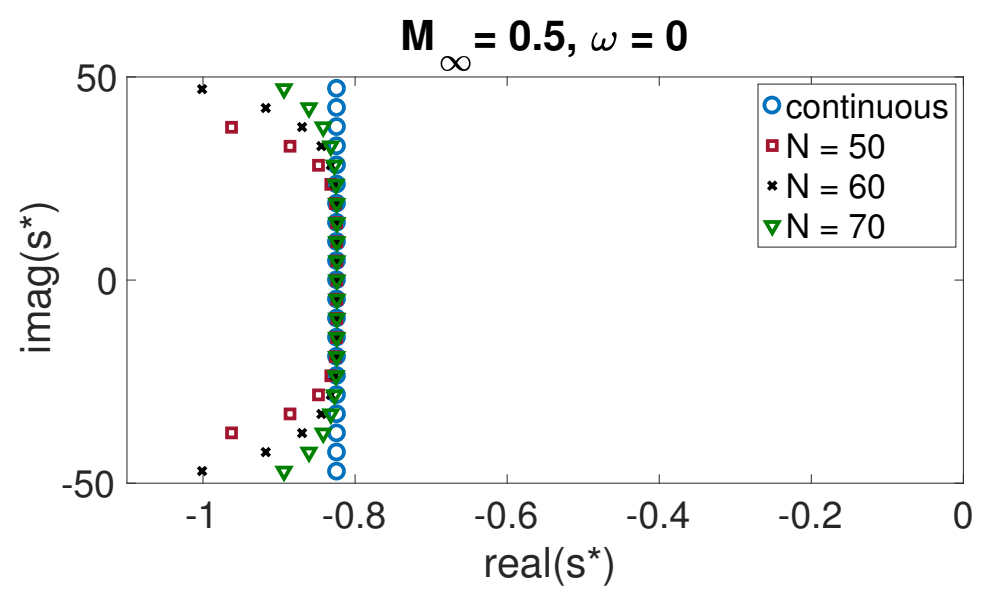

Figure 3: Comparison between the continuous and the discrete spectrum for $M_{\infty}=0.5$ and $\omega=0$.

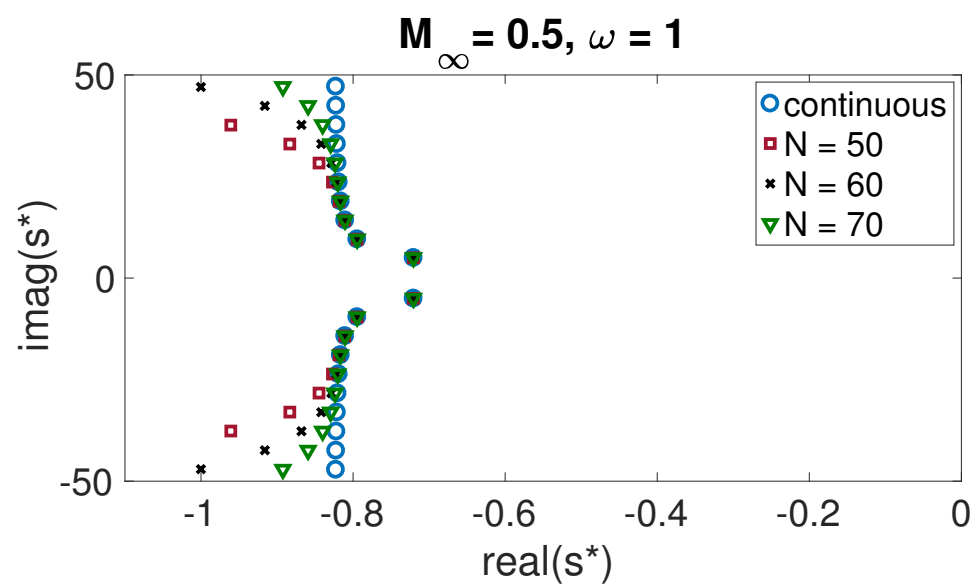

Figure 4: Comparison between the continuous and the discrete spectrum for $M_{\infty}=0.5$ and $\omega=1$. 


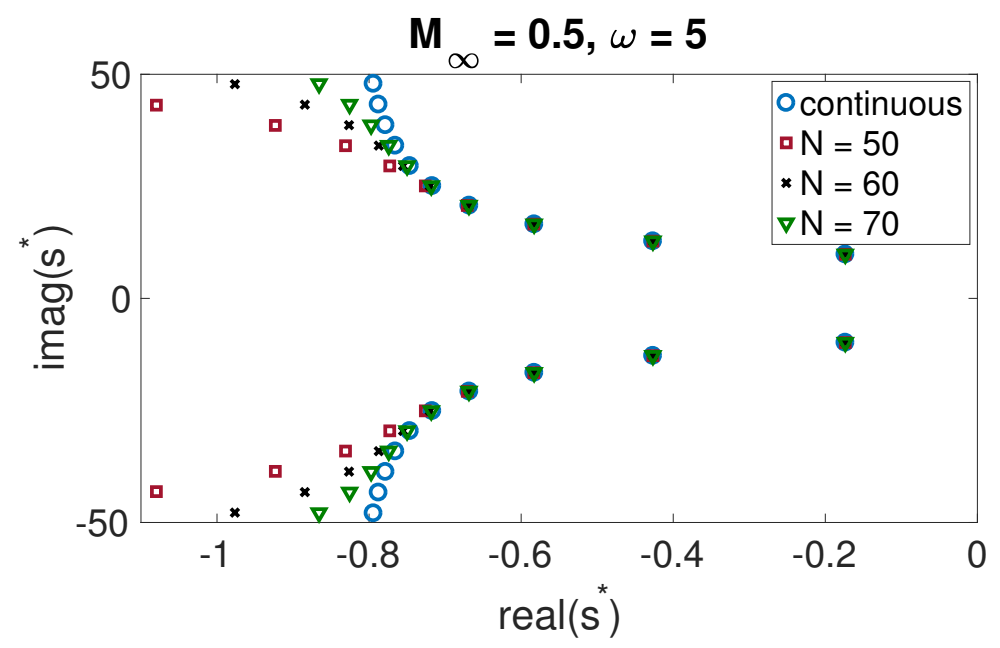

Figure 5: Comparison between the continuous and the discrete spectrum for $M_{\infty}=0.5$ and $\omega=5$.

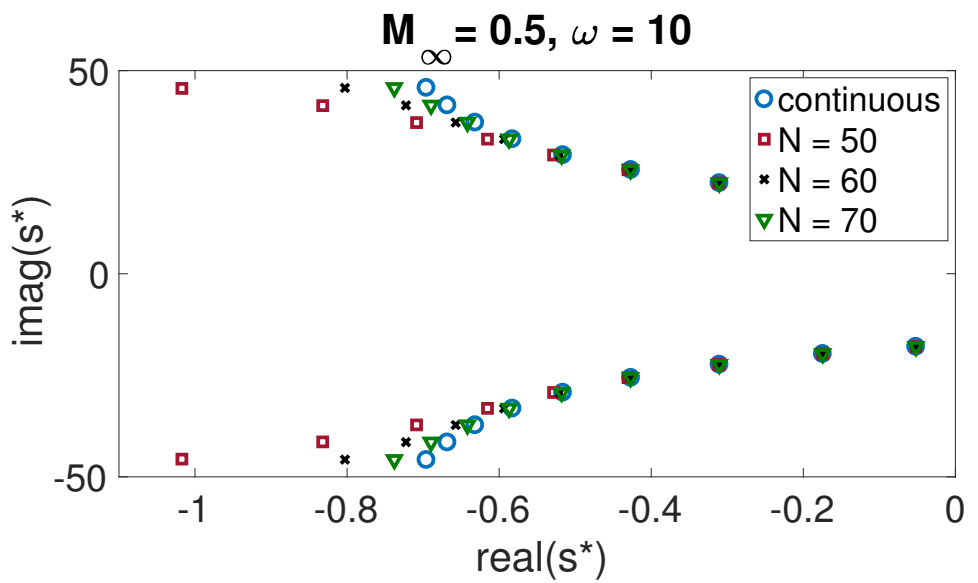

Figure 6: Comparison between the continuous and the discrete spectrum for $M_{\infty}=0.5$ and $\omega=10$. 
1) solid walls [21] and the pressure (also typically a measured quantity in experiments) is imposed at the outflow [19]. We use the nonlinear inflow conditions (2) together with (16) at the west boundary.

\subsection{The Order of Accuracy}

For verification, the method of manufactured solutions is used for the SBP-operators SBP21, SBP42, SBP63 and SBP84 with expected global convergence rate $2,3,4$ and 5 respectively [14]. The errors from the timeintegration are small enough to be neglected and the simulations are terminated at $t=5$.

A manufactured solution that satisfy the solid wall boundary conditions is

$$
\begin{aligned}
& \rho=1+0.05[\sin (\pi(x-t))+\sin (\pi(y-t))], \\
& u=1+0.05[\sin (\pi(x-t))+\sin (\pi(y-t))], \\
& v=0.05 \sin (2 \pi y) \sin (\pi x), \\
& e=3+0.05[\sin (\pi(x-t))+\sin (\pi(y-t))] .
\end{aligned}
$$

The error is measured separately for $\rho, \rho u, \rho v$ and $e$ in the $L_{2}$-norm by $\|\operatorname{err}\|^{2}=\operatorname{err}^{\top}$ Perr, where err is the pointwise error and $P$ is the quadrature corresponding to the SBP-operator. Furthermore, the convergence rate is computed as $\log \left(\|\mathbf{e r r}\|_{i} /\|\mathbf{e r r}\|_{i-1}\right) / \log \left(h_{i} / h_{i-1}\right)$, where $h$ is the gird spacing and $i$ refers to a specific number of grid points that are used in the computations. The error and rate of convergence for $\rho, \rho u, \rho v$ and $e$ are presented in Table 2, 3, 4 and 5 respectively, and agrees well with theory.

\begin{tabular}{c|cc|cc|cc|cc}
\hline Operator & \multicolumn{2}{|c|}{ SBP21 } & \multicolumn{2}{c|}{ SBP42 } & \multicolumn{2}{c|}{ SBP63 } & \multicolumn{2}{c}{ SBP84 } \\
\hline $\mathrm{N}$ & $\|$ err $\|$ & rate & $\|$ err $\|$ & rate & $\|$ err $\|$ & rate & $\|$ err $\|$ & rate \\
\hline 21 & $2.73 \mathrm{e}-04$ & - & $8.46 \mathrm{e}-05$ & - & $6.74 \mathrm{e}-05$ & - & $2.86 \mathrm{e}-05$ & - \\
41 & $6.99 \mathrm{e}-05$ & 1.97 & $1.06 \mathrm{e}-05$ & 3.00 & $2.93 \mathrm{e}-06$ & 4.53 & $1.04 \mathrm{e}-06$ & 4.79 \\
81 & $1.80 \mathrm{e}-05$ & 1.96 & $1.31 \mathrm{e}-06$ & 3.02 & $2.12 \mathrm{e}-07$ & 3.79 & $3.03 \mathrm{e}-08$ & 5.10 \\
161 & $4.65 \mathrm{e}-06$ & 1.95 & $1.63 \mathrm{e}-07$ & 3.01 & $1.44 \mathrm{e}-08$ & 3.88 & $9.39 \mathrm{e}-10$ & 5.01 \\
\hline
\end{tabular}

Table 2: Error and convergence rate for $\rho$.

\subsection{The convergence rate to steady state}

As previously mentioned, for a small initial disturbance, the solution should decay with the rate $\eta_{D}^{*}$, which in turn should converge to $\eta_{C}^{*}$ in Table 


\begin{tabular}{c|cc|cc|cc|cc}
\hline Operator & \multicolumn{2}{|c|}{ SBP21 } & \multicolumn{2}{c|}{ SBP42 } & \multicolumn{2}{c|}{ SBP63 } & \multicolumn{2}{c}{ SBP84 } \\
\hline $\mathrm{N}$ & $\|$ err $\|$ & rate & $\|$ err $\|$ & rate & $\|$ err $\|$ & rate & $\|$ err $\|$ & rate \\
\hline 21 & $3.16 \mathrm{e}-04$ & - & $9.65 \mathrm{e}-05$ & - & $6.66 \mathrm{e}-05$ & - & $3.99 \mathrm{e}-05$ & - \\
41 & $8.26 \mathrm{e}-05$ & 1.94 & $1.18 \mathrm{e}-05$ & 3.03 & $4.03 \mathrm{e}-06$ & 4.05 & $1.32 \mathrm{e}-06$ & 4.92 \\
81 & $2.21 \mathrm{e}-05$ & 1.90 & $1.44 \mathrm{e}-06$ & 3.03 & $2.83 \mathrm{e}-07$ & 3.83 & $3.58 \mathrm{e}-08$ & 5.21 \\
161 & $5.80 \mathrm{e}-06$ & 1.93 & $1.78 \mathrm{e}-07$ & 3.02 & $1.89 \mathrm{e}-08$ & 3.90 & $1.04 \mathrm{e}-09$ & 5.10 \\
\hline
\end{tabular}

Table 3: Error and convergence rate for $\rho u$.

\begin{tabular}{c|cc|cc|cc|cc}
\hline Operator & \multicolumn{2}{|c|}{ SBP21 } & \multicolumn{2}{c|}{ SBP42 } & \multicolumn{2}{c|}{ SBP63 } & \multicolumn{2}{c}{ SBP84 } \\
\hline $\mathrm{N}$ & $\|$ err $\|$ & rate & $\|$ err $\|$ & rate & $\|$ err $\|$ & rate & $\|$ err $\|$ & rate \\
\hline 21 & $1.06 \mathrm{e}-04$ & - & $8.20 \mathrm{e}-05$ & - & $6.63 \mathrm{e}-05$ & - & $1.75 \mathrm{e}-05$ & - \\
41 & $2.69 \mathrm{e}-05$ & 1.98 & $1.15 \mathrm{e}-05$ & 2.84 & $3.37 \mathrm{e}-06$ & 4.30 & $9.34 \mathrm{e}-07$ & 4.23 \\
81 & $6.75 \mathrm{e}-06$ & 1.99 & $1.42 \mathrm{e}-06$ & 3.01 & $1.72 \mathrm{e}-07$ & 4.30 & $2.93 \mathrm{e}-08$ & 4.99 \\
161 & $1.69 \mathrm{e}-06$ & 2.00 & $1.78 \mathrm{e}-07$ & 3.00 & $9.13 \mathrm{e}-09$ & 4.23 & $1.00 \mathrm{e}-09$ & 4.87 \\
\hline
\end{tabular}

Table 4: Error and convergence rate for $\rho v$.

\begin{tabular}{c|cc|cc|cc|cc}
\hline Operator & \multicolumn{2}{|c|}{ SBP21 } & \multicolumn{2}{c|}{ SBP42 } & \multicolumn{2}{c|}{ SBP63 } & \multicolumn{2}{c}{ SBP84 } \\
\hline $\mathrm{N}$ & $\|$ err $\|$ & rate & $\|$ err $\|$ & rate & $\|$ err $\|$ & rate & $\|$ err $\|$ & rate \\
\hline 21 & $9.76 \mathrm{e}-04$ & - & $2.91 \mathrm{e}-04$ & - & $2.75 \mathrm{e}-04$ & - & $8.14 \mathrm{e}-05$ & - \\
41 & $2.46 \mathrm{e}-04$ & 1.99 & $3.80 \mathrm{e}-05$ & 2.94 & $1.10 \mathrm{e}-05$ & 4.64 & $3.32 \mathrm{e}-06$ & 4.61 \\
81 & $6.17 \mathrm{e}-05$ & 1.99 & $4.76 \mathrm{e}-06$ & 3.00 & $6.11 \mathrm{e}-07$ & 4.17 & $1.05 \mathrm{e}-07$ & 4.98 \\
161 & $1.56 \mathrm{e}-05$ & 1.99 & $5.94 \mathrm{e}-07$ & 3.00 & $3.94 \mathrm{e}-08$ & 3.95 & $3.44 \mathrm{e}-09$ & 4.93 \\
\hline
\end{tabular}

Table 5: Error and convergence rate for $e$.

1. The domain in Section 4, i.e. $\Omega=[0,1] \times[-\pi, \pi]$, is considered for the computations in this section and periodic boundary conditions are imposed at $y= \pm \pi$. As initial state, $T=u=1, v=0, c=1 / M_{\infty}$ and $\rho=$ $1+0.1 e^{-(x-0.5)^{2} / 0.02} \cos (\omega y)$ and the parameters $M_{\infty}$ and $\omega$ are varied. We measure the deviation of $\rho$ from its steady-state value $\rho=1$ in the $L_{2}$-norm. Figure 7-9 presents the convergence rates for different $M_{\infty}$ and $\omega$. The SBP63 operator is used on a grid containing $80 \times 80$ grid points for all computations. The computed convergence rates agree well with the results from Section 4 and 5 . 


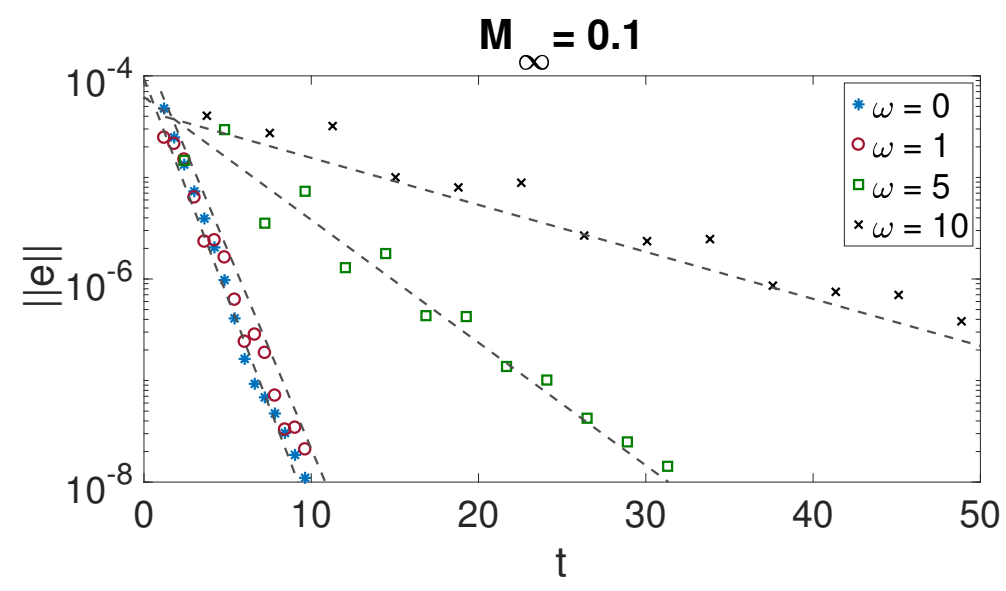

Figure 7: Comparison between the computed and theoretical convergence rates to steady state for $M_{\infty}=0.1$ and different $\omega$. The dashed lines are $\eta_{C}^{*}$ taken from Table 1.

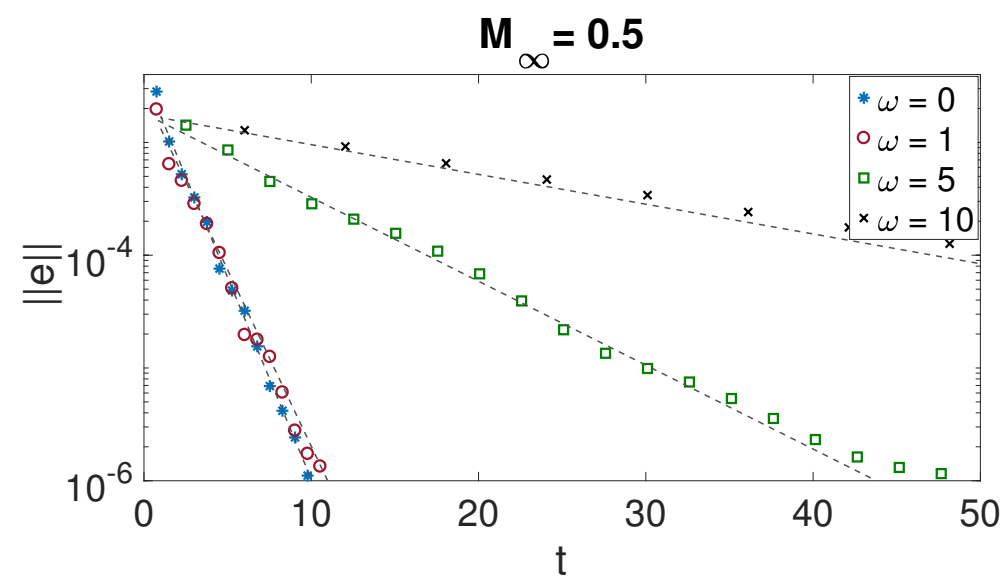

Figure 8: Comparison between the computed and theoretical convergence rates to steady state for $M_{\infty}=0.5$ and different $\omega$. The dashed lines are $\eta_{C}^{*}$ taken from Table 1 .

\section{Conclusions}

We have studied a set of practical inlet boundary conditions for internal flow calculations. Well-posedness for the imposition of the total temperature and total pressure together with the tangential velocity at the inflow boundary for subsonic flow was proved. The corresponding semi-discrete scheme was also proved to be stable. Furthermore, these inflow conditions were implemented in a nonlinear compressible Euler solver and the implementation 


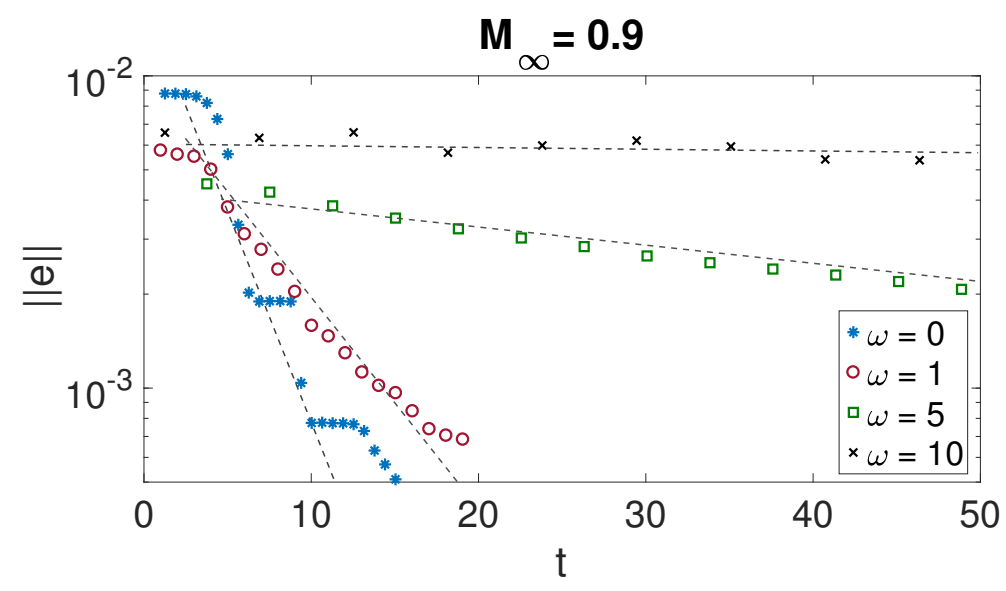

Figure 9: Comparison between the computed and theoretical convergence rates to steady state for $M_{\infty}=0.9$ and different $\omega$. The dashed lines are $\eta_{C}^{*}$ taken from Table 1.

was verified by the method of manufactured solutions. The continuous and discrete spectra were computed and the convergence rate to steady state for the inflow conditions estimated. The obtained convergence rates from the nonlinear computations agreed well with the result predicted by the linear theory.

\section{Appendix}

The relation between the linearized symmetric scheme and the fully nonlinear scheme is discussed below.

The continuous case

Let $T$ be the transformation between the conservative variables, $\vec{u}=$ $(\rho, \rho u, \rho v, e)$, and primitive variables, $\vec{v}=(\rho, u, v, p)^{\top}$, such that $\vec{u}=T \vec{v}$ and $\vec{v}=T^{-1} \vec{u}$. The Euler equations are $\vec{u}_{t}+A_{u} \vec{u}_{x}+B_{u} \vec{u}_{y}=0$. Multiplying by $T^{-1}$ from the left yields $\vec{v}_{t}+A_{v} \vec{v}_{x}+B_{v} \vec{v}_{y}=0$, where $A_{v}=M^{-1} A_{u} M$ and $B_{v}=M^{-1} B_{u} M$. The symmetric system (4) is obtained by multiplying with the symmetrizer $S^{-1}$. The transformation between the conservative and symmetric variables becomes $\vec{u}=M W$ and $W=M^{-1} \vec{u}$, where

$$
M=T S \quad \text { and } \quad M^{-1}=S^{-1} T^{-1} .
$$


Let $X$ be the matrix defined in (7) and let $q=X^{\top} W$. We obtain (8) by the relations

$$
\begin{aligned}
\left(L_{X}^{+}\right)^{-1} L W=\left(L_{X}^{+}\right)^{-1} g & \Longleftrightarrow\left(L_{X}^{+}\right)^{-1}\left(L_{X}^{+} q^{+}+L_{X}^{-} q^{-}\right)=\left(L_{X}^{+}\right)^{-1} g \\
& \Longleftrightarrow q^{+}-R q^{-}=\left(L_{X}^{+}\right)^{-1} g
\end{aligned}
$$

where $L_{X}=L X$ and $L_{X}^{ \pm}$are the columns in $L_{X}$ that correspond to $q^{ \pm}$ respectively. Further, the boundary matrix is $R=-\left(L_{X}^{+}\right)^{-1} L_{X}^{-}$.

The semi-discrete case

The transformations between the conservative variables $\overrightarrow{\mathbf{u}}$ and the symmetric variables $\mathbf{W}$ are $\overrightarrow{\mathbf{u}}=\mathbf{M W}$ and $\mathbf{W}=\mathbf{M}^{-1} \overrightarrow{\mathbf{u}}$. The transformation matrices are $\mathbf{M}=I_{x} \otimes I_{y} \otimes M$ and $\mathbf{M}^{-1}=I_{x} \otimes I_{y} \otimes M^{-1}$, where $M$ and $M^{-1}$ are defined in (30). Multiplying (25) by $\mathbf{M}$ yields

$$
\overrightarrow{\mathbf{u}}_{t}+\left(\mathbf{D}_{x} \mathbf{A}_{u}+\mathbf{D}_{y} \mathbf{B}_{u}\right) \overrightarrow{\mathbf{u}}=\mathbf{M P}^{-1} \mathbf{P}_{y}^{0} \Sigma\left(\mathbf{q}^{+}-\mathbf{R q}^{-}-\overline{\mathbf{g}}\right)+\text { MPen }
$$

where $\mathbf{A}_{u}=\mathbf{M A M} \mathbf{M}^{-1}$ and $\mathbf{B}_{u}=\mathbf{M B M}^{-1}$. Since $\mathbf{M}$ and $\mathbf{P}^{-1} \mathbf{P}_{y}^{0}$ commute, it follows from (31) and (32) that

$$
\overrightarrow{\mathbf{u}}_{t}+\left(\mathbf{D}_{x} \mathbf{A}_{u}+\mathbf{D}_{y} \mathbf{B}_{u}\right) \overrightarrow{\mathbf{u}}=\mathbf{P}^{-1} \mathbf{P}_{y}^{0} \overline{\mathbf{\Sigma}}\left(\mathbf{L}_{\mathbf{X}}^{+}\right)^{-1}(\overline{\mathbf{L}} \overrightarrow{\mathbf{u}}-\mathbf{g})+\text { MPen },
$$

where $\overline{\boldsymbol{\Sigma}}=\mathbf{M} \boldsymbol{\Sigma}$. The boundary operator is $\overline{\mathbf{L}}=\mathbf{L M}^{-1}$, where $\mathbf{L}=I_{x} \otimes I_{y} \otimes L$ is the boundary operator to the symmetric system. Lastly, we have the relation $\overline{\mathbf{g}}=\left(\mathbf{L}_{\mathbf{X}}^{+}\right)^{-1} \mathbf{g}$.

\section{References}

[1] L. Cai, J. Xiao, S. Wang, S. Gao, J. Duan, J. Mao, Gas-particle flows and erosion characteristic of large capacity dry top gas pressure recovery turbine, Energy 120 (2017) 498-506.

[2] A. Romagnoli, R. Martinez-Botas, Performace prediction of a nozzled and nozzleless mixed-flow turbine in steady conditions, International Journal of Mechanical Sciences 53 (2011) 557-574.

[3] M. Chiong, S. Rajoo, R. Martinez-Botas, A. Costall, Inlet boundary condition study for unsteady turbine performance prediction using 1-d modeling, Journal Mekanikal 53 (2011) 89-104. 
[4] M. Chiong, S. Rajoo, A. Costall, R. Martinez-Botas, Non-adiabatic pressure loss boundary condition for modeling turbocharger turbine pulsating flow, Energy Conservation and Management 93 (2015) 267-281.

[5] T. Jelinek, P. Straka, V. Uruba (Eds.), Effect of End-wall Boundary Layer and Inlet Turbulence on the Flow Field Structures in the Turbine Stage, Vol. 1745 of AIP Conference Proceedings, American Institue of Physics, 2016.

[6] N. Lamarque, T. Poinsot, Boundary conditions for acustic eigenmode computations in gas turbine combustion chambers, AIAA Journal 47 (9) (2008) 2282-2292.

[7] J. Nordström, A roadmap to well posed and stable problems in computational physics, Journal of Scientific Computing 71 (2017) 365-385.

[8] J. Blazek, Computational Fluid Dynamics: Principles and Applications, 2nd Edition, Elsevier Science, 2005.

[9] J. Anderson, Computational Fluid Dynamics: The Basics with Applications, McGraw-Hill Education, New York, 1995.

[10] B. Gustafsson, H.-O. Kreiss, J. Oliger, Time Dependent Problems and Difference Methods, John Wiley \& Sons, Inc., 1995.

[11] J. Nordström, The influence of open boundary conditions on the convergence to steady state for the Navier-Stokes equations, Journal of Computational Physics 85 (1989) 210-244.

[12] J. Nordström, S. Eriksson, P. Eliasson, Weak and strong wall boundary procedures and convergence to steady-state of the Navier-Stokes equations, Journal of Computational Physics 231 (2012) 4867-4884.

[13] H.-O. Kreiss, J. Lorentz, Initial Boundary Value Problems and the Navier-Stokes equations, SIAM, 1989.

[14] M. Svärd, J. Nordström, Review of summation-by-parts schemes for initial-boundary-value problems, Journal of Computational Physics 268 (2014) 17-38.

[15] N. Hall, Isentropic flow, https://www.grc.nasa.gov/www/BGH/ isentropic.html, online: accessed 8 Jan 2018 (2015). 
[16] B. Gustafsson, High Order Difference Methods for Time Dependent PDE, Springer-Verlag Berlin Heidelberg, 2008.

[17] H. Lomax, T. H. Pulliam, D. Zingg, Fundamentals of Computational Fluid Dynamics, Springer, Berlin, 2001.

[18] S. Abarbanel, D. Gottlieb, Optimal time splitting for two - and threedimensional Navier-Stoeks equations with mixed derivatives, Journal of Computational Physics 31 (1981) 1-33.

[19] M. Svärd, J. Nordström, A stable high-order finite difference scheme for the compressible Navier-Stokes equations, far-field boundary conditions, Journal of Computational Physics 225 (2007) 1020-1038.

[20] B. Engquist, B. Gustafsson, Steady state computations for wave propagation problems, Mathematics of Computations 179 (1987) 39-64.

[21] J. Berg, J. Nordström, Stable Robin solid wall boundary conditions for the Navier-Stokes equations, Journal of Computational Physics 230 (19) (2011) 7519-7532. 